\title{
El valor de la comunicación estratégica para la gestión responsable y la prevención de conflictos mineros $^{1}$
}

\section{The value of strategic communication for accountability and conflict prevention miners}

Lic. Sandra CARRILLO HOYOS Pontificia Universidad Católica del Perú (Perú) sandra.carrillo@pucp.pe

Recibido: 10 de septiembre 2013 Aceptado y Publicado: 18 de noviembre de 2013

\section{Resumen}

En Perú, los conflictos entre empresas mineras y comunidades locales son cada vez más visibles y relevantes para la opinión pública debido al incremento de las situaciones de crisis registradas en los últimos años. Dadas las dificultades para el entendimiento mutuo entre los actores, se ha constituido como un asunto de estudio de la comunicación para el desarrollo. Partiendo de esta problemática, se realizó un estudio sobre los modelos y prácticas comunicativas que se desarrollan entre las empresas mineras y las comunidades locales en conflicto. A partir de la metodología de estudio de casos, se seleccionaron tres que recorren distintas etapas de relacionamiento, cuyo análisis comparativo contribuya a la comprensión general de las situaciones de conflicto en el país.

El presente artículo, además de presentar los resultados del mencionado estudio, propone un modelo de intervención comunicativa para la prevención de conflictos, incorporando la responsabilidad social corporativa como un modelo de gestión y reconociendo el valor estratégico del relacionamiento coherente con todos los grupos de interés. En este marco, la comunicación ha dejado de ser concebida como una herramienta empleada con fines utilitarios, para revalorar su potencial estratégico, desempeñando un rol fundamental desde la identificación y mapeo de los grupos de interés, hasta el diseño, implementación y monitoreo de la estrategia de relacionamiento con cada uno.

\footnotetext{
${ }^{1}$ Artículo producto de una Ponencia presentada en el VI Encuentro Panamericano de Comunicación COM PANAM 2013 celebrado la Escuela de Ciencias de la Información de la Universidad Nacional de Córdoba (Argentina), durante los días 5, 6 y 7 de junio de 2013.
} 


\begin{abstract}
In Peru, the conflict between mining companies and local communities are becoming more visible and relevant to the public due to increasing crises in recent years. Given the difficulties of mutual understanding between actors, it has been established as a matter of study of communication for development. Based on this problem, I conducted a research on the patterns and communication practices developed between mining companies and local communities in conflict. From the case study methodology, three were selected wich cover different stages of relationships, whose comparative analysis contributes to the general understanding of conflict situations in the country.

This article, besides presenting the results of this study, proposes a model of communication intervention for conflict prevention, incorporating corporate social responsibility as a management model and recognizing the strategic value of consistent relationships with all stakeholders. In this context, the communication is no longer conceived as a tool used for utilitarian purposes, to reassess its strategic potential, playing a key role from the identification and mapping of stakeholders, to design, implementation and monitoring of the strategy of relating to each.
\end{abstract}

Palabras Clave: conflicto, minería, prácticas comunicativas, asimetrías, relaciones de poder y responsabilidad social.

Key Words: conflict, mining, communication practices, asymmetries, power relations and social responsibility.

\title{
1. Introducción
}

En las últimas décadas, América Latina se ha convertido en una de las regiones más importantes del mundo para atraer inversiones en minería. Si bien ello ha generado un impacto macroeconómico positivo en la región, también ha generado la multiplicación de 
conflictos sociales entre las empresas mineras y las comunidades aledañas a sus operaciones.

En Perú, los conflictos entre empresas mineras y comunidades locales han cobrado visibilidad por el incremento cuantitativo de los casos de crisis y la intensidad de la violencia ejercida por los actores involucrados en los últimos años. Esta problemática ha puesto en evidencia la ausencia de mecanismos eficaces de prevención y gestión de conflictos en el ámbito estatal, empresarial y de la sociedad civil.

Si bien cada caso de conflicto obedece a un contexto particular del relacionamiento entre la empresa y la comunidad, en el cual convergen aspectos sociales, económicos y ambientales, es posible reconocer detonantes recurrentes de crisis como: la desconfianza, la desinformación, la construcción de prejuicios y la incertidumbre. Dichos problemas señalan que el enfoque comunicativo puede aportar en el análisis de la problemática de conflictos y en la implementación de propuestas que apunten a construir confianza, promuevan el diálogo sincero, y construyan espacios que permitan establecer planes de desarrollo concertados entre la empresa minera y la comunidad local.

A partir de ello, la investigación realizada buscó caracterizar las prácticas comunicativas que se desarrollaban en situaciones de conflicto entre empresas de mediana minería (área de concesión mayor a 2,000 ha y capacidad productiva entre 350 y 5,000 TMD) y comunidades locales ubicadas en la sierra central (población dedicada a actividades agropecuarias, con acceso limitado a servicios básicos y alto nivel de pobreza).

Siendo actores tan diferentes en cuanto a su definición, manejo de información, recursos económicos y respaldo legal y político, la empresa minera y la comunidad local entablan una relación social porque comparten el espacio y ciertos recursos naturales. En este relacionamiento, se observaron las "prácticas comunicativas", que engloban todos los procesos de interacción social emprendidos de forma consciente o inconsciente. 
Las prácticas comunicativas se desarrollaban sobre la base de "situaciones de conflicto", entendidas en el estudio como una serie de acontecimientos organizados alrededor de las diferencias construidas por las posiciones y acciones de la empresa minera y la comunidad local a lo largo de su relacionamiento (De Echave, 2009). Las fricciones entre las partes van agravando la hostilidad hasta que sus posiciones y exigencias resultan irreconciliables, originando eventos de crisis y violencia.

Entonces, es posible que el conflicto de intereses entre una empresa minera y una comunidad local sea permanente; sin embargo, su duración e intensidad dependerán del desarrollo del relacionamiento, así como de las actitudes y comportamientos de los actores. Por tal motivo, esta investigación abarcó el análisis a profundidad de tres casos que recorrían distintas etapas de conocimiento, interrelación y conflicto.

En el presente artículo, se parte de los resultados de la investigación realizada y los hallazgos alrededor de las condiciones necesarias para el entendimiento mutuo entre la empresa minera y la comunidad, para plantear un modelo de intervención comunicativa para la prevención de conflictos. Dicho modelo se origina en el reconocimiento de las oportunidades de acción de la empresa minera a través de su gestión de la responsabilidad social corporativa, para construir relaciones de confianza con los grupos de interés en los que genera un impacto.

\section{Metodología}

Se buscó, en primer lugar, explorar las características que definen a las empresas mineras medianas y a las comunidades locales de la sierra central del Perú a partir de sus prácticas culturales y económicas, visiones de desarrollo y proyecciones a futuro, organización y prácticas comunicativas para la toma de decisiones. El segundo objetivo estuvo orientado a describir el contexto en el que se comunican, sobre la base del conocimiento de la historia de 
la relación, las percepciones conflictivas y las expectativas sobre el otro desarrolladas durante la convivencia, la situación actual de la relación y el conflicto de intereses. Finalmente, el tercer objetivo consistió en comprender el proceso social de comunicación, con el fin de identificar los factores que dificultan el entendimiento entre la empresa minera y la comunidad local para poder reconocer las situaciones que pueden mejorarse o prevenirse.

\subsection{Estudio de casos}

La investigación del estudio constituyó un estudio de cualidades y rasgos en las prácticas sociales de comunicación que se desarrollan en el relacionamiento entre empresas mineras y comunidades locales. En este universo se empleó la metodología de estudio de casos; concretamente, se seleccionaron tres empresas mineras medianas y comunidades locales ubicadas en la sierra central del Perú, cuyo estudio a profundidad y posterior comparación permitiera contribuir a la comprensión más general de la problemática.

La segmentación de empresas mineras medianas ${ }^{2}$ obedeció a que constituyen un nivel intermedio de complejidad, debido al tamaño y nivel organizativo de sus integrantes; y la elección de comunidades ubicadas en la sierra central se debió a que ésta es, ancestralmente, una zona minera donde se puede encontrar los vestigios de esta naturaleza más antiguos del país. Los casos seleccionados fueron contrastables por recorren distintas etapas de relacionamiento ${ }^{3}$.

El primero comprende al Proyecto Mallay, ${ }^{4}$ de la Compañía de Minas Buenaventura, y a la comunidad del mismo nombre ${ }^{5}$, ubicada a 3.500 msnm en el distrito y provincia de Oyón,

\footnotetext{
${ }^{2}$ Una empresa minera mediana se define como una concesión cuya área abarca más de 2.000 hectáreas y tiene una capacidad de producción entre 350 toneladas métricas y 5.000 toneladas métricas diarias (OSINERGMIN 2010).

3 Para su selección se siguieron criterios como la accesibilidad, la disposición de los actores involucrados, el grado de complejidad de la relación y los años de relacionamiento (Stake 1998).

${ }^{4}$ El proyecto minero se inició en el 2005 y proyectó producir 500.000 tm de oro, plata, plomo y zinc.
} 
departamento de Lima. Entre las aristas más interesantes de este caso se encuentra que la comunidad se generó expectativas laborales acerca de la presencia de la empresa en la zona, lo que obedeció a una diferencia en las percepciones sobre los acuerdos del periodo de consulta entre las partes, realizado entre los años 2005 y 2007.

A 5 años de relacionamiento, se encontró que el proyecto minero había despedido a buena parte del personal obrero local, lo que había generado rivalidad y competencia al interior de la comunidad. Esta última, a su vez, se caracterizaba por no tener objetivos claros y armónicos, y, además, por sus problemas de representatividad.

El segundo caso estudia la relación entre la Unidad Minera Iscaycruz, de la Empresa Minera Los Quenuales ${ }^{6}$, y la Comunidad Campesina de Curay ${ }^{7}$, ubicada en el distrito de Pachangara, provincia de Oyón, también a 3.500 msnm. La historia de la relación se remonta al año 1992, cuando la empresa solicitó permiso para la instalación de un mineroducto en la comunidad, previa consulta con las autoridades. Al aceptar la solicitud, la comunidad se generó falsas expectativas sobre las posibilidades de trabajo en la empresa o respecto de posibles negocios con ella.

A esta frustración de expectativas se añadió luego, para agravar el panorama, el daño causado a los canales de riego de la comunidad durante la instalación del mineroducto, que afectó la actividad agrícola. Solo en el año 2003, con el cambio de administración de la Unidad Minera por la empresa Los Quenuales ${ }^{8}$, se estableció un convenio por el que la empresa se comprometía a emplear a los comuneros en el trabajo de reparación de la tierra

\footnotetext{
${ }^{5}$ La comunidad de Mallay tiene 502 habitantes. La población se dedica a la ganadería y la agricultura para el autoconsumo.

${ }^{6}$ La unidad minera inició operaciones en el año 1996 bajo la administración de la Empresa Minera Iscaycruz para producir plomo y zinc.

${ }_{77}$ Cuando se visitó la zona la comunidad de Curay tenía aproximadamente 326 habitantes, quienes se dedicaban a la agricultura y la ganadería para el autoconsumo.

${ }^{8}$ Debido a que Los Quenuales incorpora la inversión de Glencore International, empieza a adoptar una serie de lineamientos para el relacionamiento con las comunidades de su entorno.
} 
y los canales ${ }^{9}$. Si bien la puesta en práctica de este convenio mejoró la economía familiar, a su vez, generó otros problemas como la dependencia de la comunidad para con la empresa.

Finalmente, el tercer caso concierne a la relación entre la Unidad Huarón, de la Empresa Minera Pan American Silver ${ }^{10}$, y la Comunidad Campesina de Huayllay, ubicada en el distrito del mismo nombre ${ }^{11}$, provincia y región de Pasco, entre los 4.100 y los $4.850 \mathrm{msnm}$. Esta relación tiene un origen muy antiguo, pues se inició en 1912, con la instalación de la Compañía French Penarroya para constituir la mina Huarón.

Cien años después, encontrarse con que Huayllay es considerado un "distrito minero" por la residencia de trabajadores de este ramo en la zona urbana, así como por la oferta de servicios relacionados con la minería como fuente importante de ingresos, podría indicar que no existe conflicto alguno con la empresa minera. Sin embargo, el análisis de la historia de la relación permite comprender que la población originaria, a falta de oportunidades laborales y ante el deterioro ambiental provocado por la minería, o se trasladó a otras regiones o se quedó en los caseríos rurales, al tiempo que mucha población de otras regiones se mudaba al distrito para trabajar en la mina.

La visita al lugar y los testimonios de la población de los caseríos dan cuenta de que ésta sigue siendo pobre a pesar de 100 años de minería. Su descontento se profundiza cuando percibe las posibilidades de las que ha gozado la población migrante, que se ha asentado en el distrito apenas hace 30 años.

\footnotetext{
${ }^{9}$ Los comuneros recibían una remuneración diaria de 35 soles y eran supervisados por una empresa contratista.

10 Cuando se realizó el trabajo de campo, la Unidad Huarón tenía aproximadamente 1.500 trabajadores y se dedicaba a la producción de cobre, plata, plomo y zinc.

${ }^{11}$ Cuando se realizó el trabajo de campo, el distrito de Huayllay tenía 13.000 habitantes entre el centro urbano y 6 caseríos, dedicados al trabajo en las empresas mineras, ganadería, turismo y, en menor medida, la artesanía textil.
} 


\subsection{El recojo de información}

Las variables del estudio fueron agrupadas en cuatro temas, que responden a los objetivos específicos de la investigación: la caracterización general de cada actor (empresa y comunidad por separado), las prácticas comunicativas y organizativas al interior de cada uno, el contexto de la relación entre ambos y el proceso comunicativo que se desarrolla en el relacionamiento entre la empresa y la comunidad.

Tomando en consideración las variables seleccionadas para el estudio, se identificaron unidades de observación y fuentes de información para llevar a cabo el estudio de cada caso. Las técnicas de acopio de información primaria utilizadas fueron: entrevistas a profundidad, grupos focales y observación.

\section{Resultados}

En Perú, la relación entre empresas mineras y comunidades locales ha estado marcada en los últimos años por una situación tensa que en no pocos casos ha derivado en enfrentamientos violentos que han dificultado las posibilidades de diálogo.

Es lamentable reconocer que la inadecuada gestión de los conflictos ha generado grandes pérdidas para el país: entre los años 2006 y 2012, 219 personas murieron y 2,949 fueron heridas durante los conflictos sociales, gran parte de ellos relacionados a la actividad extractiva (Defensoría del Pueblo, 2013).

En el análisis de la problemática desde diferentes perspectivas en los últimos años, se ha señalado que el incremento de los casos de conflicto y la intensidad ejercida se deben a una "falta de comunicación" entre los involucrados. Sin embargo, partiendo de la definición de comunicación como un proceso social cuyo objetivo es el entendimiento mutuo de los interlocutores -- quienes a su vez son sujetos de intenciones, motivaciones y habilidades -(West, 2005), así como de las características de los actores involucrados y el contexto en el 
que se relacionan, ¿es realmente esperable que exista diálogo?, ¿hasta qué punto se puede intervenir desde la comunicación y qué aporte puede ofrecer esta perspectiva?

Como primera premisa se encuentra que, si bien los actores involucrados, la empresa y la comunidad, son formalmente pares, en la práctica uno tiene una serie de ventajas sobre el otro, debido a la inequidad económica, política y social que favorece una relación de poder entre ellos.

Dado el poder adquirido por el sector privado en las economías del mundo, como resultado del modelo de desarrollo hegemónico basado en el mercado, la empresa privada ha dejado de constituir un actor económico individual y se ha convertido en un actor social que se relaciona como tal con el Estado y la sociedad civil. En el caso específico del Perú, la minería es el sector con mayor poder, pues el país se ha convertido en uno de los principales receptores de inversión minera en la región América Latina y el Caribe (Centro de Estudios del Cobre y la Minería, 2012).

Desde mediados de la década de 1990, y luego de la implementación de las reformas de ajuste estructural y de las políticas de atracción de capitales extranjeros, el país ha experimentado un explosivo crecimiento de la actividad minera (De Echave, 2009). Estas medidas generaron algunos cambios en el rol del Estado en la economía nacional, entre los que destaca la transferencia de empresas y proyectos mineros al sector privado. Si bien cruciales, las reformas legales no fueron los únicos elementos en el proceso de atraer inversionistas extranjeros. Después de una década perdida por el colapso económico y la violencia social (1980-1990), fue también necesario crear una imagen del Perú como "un lugar donde hacer negocios" (Damonte, 2006).

Estos esfuerzos hicieron posible un enorme crecimiento de la inversión minera, que superó los 10 mil millones de dólares entre los años 1992 y 2007, y le otorgaron al Perú una imagen internacional de distrito minero, particularmente de oro y cobre. En la actualidad el país es considerado uno de los principales productores de plata, oro, cobre y zinc (Consejo 
Internacional de Minería y Metales, 2013). Esto significó, además, un rápido avance territorial, pues a mayo de 2013 se han concedido 26 millones de hectáreas (más del 21\% del área total del Perú) a la actividad minera (Cooperacción, 2013).

Sin embargo, la mayoría de comunidades afectadas por la minería ha sido muy poco beneficiada por el crecimiento económico y la expansión del sector. La ausencia de oportunidades para el desarrollo, en el marco de un modelo económico orientado básicamente a la exportación de materia prima, ha permitido la formación de enclaves de pobreza en las comunidades locales afectadas por la minería, lo que ha incrementado progresivamente el descontento social de la población. Así, las tensiones acumuladas en la historia de la relación han facilitado las situaciones de conflicto entre empresas mineras y comunidades locales.

Es cierto, entonces, que las situaciones de crisis que marcan el conflicto suelen ser reforzadas por la poca disposición de los actores para comunicarse entre sí (Cedrón, 2007), pero esto no oculta que el origen del problema se encuentra en la confluencia de intereses opuestos y la ausencia de condiciones para el diálogo. De lo que se desprende que, si bien la comunicación no se encuentra en la base del problema, puede aportar significativamente en la construcción de las condiciones para el diálogo y en la reducción de la inequidad entre los actores a través del fortalecimiento del actor social comunidad local (Gumucio, 2008).

Para argumentar esta propuesta se desarrollan a continuación tres segmentos: la caracterización de los actores involucrados en el conflicto, el contexto actual en el que se relacionan y la aplicación del enfoque integral de la comunicación al relacionamiento entre empresas mineras y comunidades locales. 


\subsection{Caracterización de los actores en conflicto}

Se ha identificado cuatro grandes categorías de actores, cada uno de ellos con racionalidades, intereses y canales de comunicación particulares. Las empresas mineras, las comunidades locales y el Estado aparecen como centrales y configuran un triángulo de interacción que define las características de los conflictos. A ellos se suman algunos actores extralocales que, dependiendo del caso, intervienen en la dinámica del conflicto.

\subsubsection{La empresa minera}

Sea cual fuere su capacidad productiva, la mayoría de empresas mineras en el Perú se orientan a la exportación de materia prima. A pesar del impacto macroeconómico de esta actividad, los niveles de encadenamiento productivo y el escaso uso de mano de obra local hacen que la minería aporte muy poco a las economías locales (Barrantes 2005).

Entre los casos estudiados, la comunidad de Huayllay es emblemática, pues a pesar de la sostenida actividad minera durante los últimos cien años, su población no ha logrado cubrir las necesidades básicas para mantener una calidad de vida digna.

El descontento de las comunidades poco beneficiadas por el crecimiento de la minería, como en el mencionado caso, ha despertado en las empresas del sector la necesidad de contar con una "licencia social para operar", con el fin de evitar problemas con el entorno que pudieran interrumpir o dificultar sus actividades (Alayza 2007). Esta necesidad, sumada al crecimiento de las brechas sociales durante los años del boom minero y al auge internacional de la responsabilidad social empresarial, ha traído como consecuencia que las empresas mineras se preocupen por mantener buenas relaciones con las comunidades aledañas a su operación (De Echave 2009).

Sin embargo, esta preocupación se ha traducido en la implementación de iniciativas que, en lugar de promover el desarrollo local, suelen apuntar a minimizar el descontento de la 
población del lugar, a la que buscan convencer de los beneficios que trae la actividad minera para, así, asegurar la eficiencia económica, ambiental y social de la minería corporativa actual.

De este modo, parte de las acciones de responsabilidad social y relaciones públicas ha estado orientada a disminuir las tensiones existentes y a reducir la eventualidad de una protesta. Cuando, aun así, ella ocurre, la empresa se ve en la necesidad de aquietarla con acciones que oscilan entre el control de la situación, la participación en mesas de diálogo y la implementación de campañas para generar una opinión pública favorable a su posición (De Echave 2009).

De ahí que, si bien la responsabilidad social y relaciones públicas deberían promover que la empresa mantenga un óptimo relacionamiento con sus grupos de interés, en la práctica ambas han sido utilizadas por buena parte de las empresas mineras como estrategia para mitigar los conflictos con la comunidad local, en lugar de atacar el fondo de éstos o contribuir al desarrollo local. Por ello, en los tres casos tratados en el estudio, este interés por mantener una buena imagen social externa no se condice con las dificultades de estas organizaciones para comunicarse con los trabajadores obreros, a pesar de que constituyen la mayor parte de su personal y pertenecen a la comunidad aledaña a la zona de extracción.

\subsubsection{La comunidad local}

Las comunidades locales son los actores más afectados por el proceso de convivencia con la empresa minera, debido a la inevitable alteración de sus modos tradicionales de vida a causa de la explotación del territorio donde habitan. Se trata por lo general de poblaciones rurales dedicadas a actividades agropecuarias, que se organizan en diversas asociaciones de pobladores y productores (De Echave 2009). 
La forma más frecuente es la comunidad campesina, organización de la sociedad civil que representa a la comunidad local ante la empresa minera no solo en el plano de la interacción social, sino también en el marco de la negociación legal por el uso y control del territorio superficial que administra. A pesar de que constituye la forma de organización más antigua en el espacio rural peruano, y de que han transcurrido casi 90 años desde su reconocimiento legal, la situación de vulnerabilidad jurídica y social de las comunidades campesinas no ha cambiado lo suficiente (Castillo, P. 2007). Prueba de ello es la existencia de más de 6 mil comunidades campesinas legalmente reconocidas en las que se encuentran los más altos índices de pobreza y de pobreza extrema del Perú (Grupo ALLPA 2010: 3).

Nada de esto basta, sin embargo, para que estas comunidades sean priorizadas por las políticas públicas y resulten visibles para la ciudadanía. La falta de interés oficial obedece al modelo de desarrollo adoptado por los últimos gobiernos, basado en la promoción de la inversión minera en el sector rural y en la exclusión de la población campesina. Asimismo, frente a la teoría sobre la forma de organización tradicional andina, el espacio rural contemporáneo no está necesariamente articulado y sus miembros difícilmente cuentan con intereses claros y armónicos (Diez 2007b). Más aún: su visión de desarrollo es ambigua, e integra el modelo basado en el mercado y las características de la racionalidad andina (Sánchez 1982). ${ }^{12}$

A través de los casos estudiados se pudo constatar que, cuando llegó a la comunidad, la empresa minera tenía bien definido su objetivo, en tanto las tres comunidades estudiadas (Mallay, Curay y Huayllay) carecían de intereses colectivos y las relaciones entre sus miembros eran poco armónicas. Esta situación resulta particularmente interesante en el caso de Mallay. Allí la comunidad inició la relación con la empresa sin un interés que involucrara a la mayor parte de los pobladores. Como cada familia veía únicamente por sus propios

\footnotetext{
12 No existe un "modelo" de desarrollo andino conceptualizado como tal, pero sí una racionalidad andina que ha sido estudiada y que sirve de base para comprender la lógica económica y de organización de los modos de vida rurales (Sánchez 1982).
} 
intereses, se generó rivalidad y competencia por acceder a las oportunidades que brinda el proyecto minero. Esto hizo de Mallay una población fácil de dividir y, por ende, débil frente a actores externos. Así, pues, no fue la llegada del proyecto minero lo que provocó la división actual de la población, aunque sí la profundizó y se benefició de esta debilidad.

Resulta de lo anterior que un factor común a los tres casos es la debilidad institucional de la comunidad campesina como organización civil local y que, en teoría, debería representar los intereses de la población ante la empresa minera. En los tres casos se han encontrado problemas de representatividad, personajes haciendo de delegados de la organización comunal sin serlo realmente.

En relación con estos problemas de representatividad y liderazgo se encontró también que, al no existir espacios ni canales efectivos de comunicación al interior de la comunidad, la información se concentraba en las autoridades, lo que generaba rumores e incertidumbre.

Además, se constató en los tres casos una suerte de desinterés de la población local por participar de las organizaciones, informarse o calificar para tener voz y voto en la toma de decisiones de la comunidad campesina. Se encontró entonces que las colectividades locales se enfrentaban a la problemática desde una posición estructural de debilidad económica, técnico-legal y social.

\subsubsection{El Estado}

Desde 1990, los sucesivos gobiernos han optado por realizar una serie de reformas legales - como la privatización del sistema de propiedad de la tierra - y económicas —como los incentivos tributarios - destinadas a facilitar las inversiones orientadas a la explotación de los recursos mineros (De Echave 2009). Así mejoró el desempeño de la economía nacional, que había decaído severamente entre la segunda mitad de la década de 1970 e inicios de la de 1990, y este impacto positivo marcó el inicio del discurso minero como solución a la 
pobreza (Damonte 2006). Sin embargo, la mayor recaudación del Estado gracias a los tributos pagados por las empresas mineras no ha sido gestionada eficientemente. $Y$ eso explica que el crecimiento macroeconómico no se haya traducido en una reducción de la pobreza en las comunidades afectadas por la minería.

El ingreso por canon minero ${ }^{13}$ debería invertirse en bienes de capital que sirvan para generar el desarrollo de la población local (Francke 2002), pero esto demanda, fundamentalmente, una efectiva administración y distribución de los recursos, que dependen, a su vez, de las capacidades de las instituciones locales. Ocurre, sin embargo, que en muchos casos ellas no están preparadas para llevar a cabo una gestión eficiente, o que están atravesadas por la corrupción, lo que trae como consecuencia que no inviertan de manera adecuada los recursos que reciben.

Ésta es una de las principales razones por las cuales las provincias donde se desarrolla actividad minera figuran entre las más pobres del país, por debajo del promedio nacional (Barrantes 2005). El divorcio entre la situación local y el crecimiento macroeconómico nacional ha generado en las poblaciones de las zonas mineras la pérdida de credibilidad del Estado para la gestión de los conflictos socioambientales (Aste 2004b). En la práctica, la participación del Estado se ha dirigido más a resolver los problemas generados por los estallidos de los conflictos que a identificar y atacar las causas que los generan. Lejos, entonces, de actuar con firmeza, autonomía y credibilidad, y de preocuparse por los problemas sociales y ambientales (Barrantes 2005), el Estado se limita a mantener el orden público para evitar que las protestas desalienten la inversión minera (De Echave 2009).

${ }^{13}$ El canon minero consiste en la repartición a los gobiernos regionales y los gobiernos locales de las jurisdicciones donde se encuentran los yacimientos mineros del total de ingresos y rentas obtenidos por el Estado por la explotación económica de esos recursos naturales. Estos impuestos representan aproximadamente el 50\% del total de ingresos y rentas obtenidos por el Estado en la actividad minera por el aprovechamiento de los recursos minerales, metálicos y no metálicos (Ley de Canon 27506, artículo 8.). 


\subsubsection{Los actores extralocales}

Es importante resaltar la ausencia de actores extralocales con poder en los tres casos estudiados. No se ha registrado una presencia significativa de las ONG, organismos de concertación y organizaciones de la sociedad civil externas a las comunidades, que podrían mediar e influir positiva o negativamente en la relación.

Tampoco se ha encontró medios locales de comunicación en Mallay y Curay. Como las dos son comunidades pequeñas y pobres que aún no han cubierto sus necesidades básicas, los pobladores y las autoridades no ven la comunicación como un asunto prioritario. Ello hace que ambas comunidades se encuentren mucho menos informadas de lo que sucede en el país y sean más susceptibles de ser movilizadas por agentes externos.

En el caso de Huayllay, donde sí existía una radio local y un canal de televisión por el que el Alcalde transmitía mensajes, estos espacios eran financiados por las empresas mineras o por el Concejo Municipal. Ello explicaba que los programas que se transmiten en esa localidad mantengan una visión favorable a la inversión minera.

\subsection{Contexto del relacionamiento entre la empresa y la comunidad}

Los conflictos entre empresas mineras y comunidades locales pueden entenderse como una serie de acontecimientos organizados alrededor de las diferencias entre ambas, generados por las posiciones y acciones sostenidas por cada una a lo largo de la relación. Estas diferencias de posiciones y objetivos provocan continuas tensiones que eventualmente desembocan en enfrentamientos que pueden demandar la intervención del Estado para la recuperación del orden público, de agencias especializadas para la resolución de conflictos y de otros actores externos que actúan a favor de alguna de las partes (De Echave 2009). 
Las referidas situaciones de crisis son resultado de un proceso de acumulación de fricciones entre la empresa y la comunidad que van incrementando la hostilidad hasta que sus posiciones y exigencias resultan irreconciliables. Según esta lógica, el conflicto de intereses entre una empresa minera y una comunidad local puede ser permanente; sin embargo, su duración e intensidad dependerán del tratamiento de la relación, así como de las actitudes y comportamientos de los actores.

Una de las premisas más importantes en la mayoría de conflictos mineros reside en las preocupaciones medioambientales; preocupaciones que se encuentran presentes en los casos de estudio. Aun así, el componente ambiental muy pocas veces es el más fuerte. Con muy poca frecuencia la defensa de los recursos naturales reivindica su valor propio, a pesar de que éste es por lo general el discurso que se propaga hacia la opinión pública.

En las comunidades estudiadas el ambiente es valorado en relación con el derecho de acceso a la tierra y el agua, sustento de la economía familiar. En el caso de Curay, por ejemplo, lo que estaba en juego era el recurso agua de la comunidad, cuyo uso fue perjudicado por la instalación del mineroducto, que provocó daños a los canales de regadío. Como se puede ver, la causa del problema no era la preocupación de la comunidad por el recurso en sí mismo, sino porque lo necesitaban para desarrollar sus actividades agropecuarias, sustento económico de las familias que allí viven.

Por eso fue interesante encontrar que en ninguno de los casos estudiados había grupos de pobladores en contra de la minería porque, por ejemplo, podría contaminar el agua, el aire o el suelo de sus localidades. Las tres comunidades se encontraban a favor de la inversión minera y aspiraban a conseguir trabajo en las unidades mineras o a constituir algún negocio familiar relacionado con la mina. De esta forma, en los tres casos materia del estudio, el conflicto entre la empresa minera y la comunidad tenía un fuerte componente económico, no siempre detectado por las partes ni atacado efectivamente para alcanzar el mutuo beneficio. 
En los tres, además, se encontró que la empresa minera se instaló en un contexto con poco o nulo conocimiento y experiencia sobre minería. En esa primera etapa, las mineras realizan exploraciones en la comunidad, hasta que se alcanza una aproximación a la cantidad y calidad de mineral. Si el resultado es positivo, la instalación de la unidad minera (o de un mineroducto) se somete a consulta en la comunidad. Lo más común es que luego del proceso de consulta la comunidad acepte la instalación, debido a las expectativas sobre los beneficios que puede traer a la población, especialmente relacionados con oportunidades de trabajo.

En Curay y Mallay, transcurridos algunos años desde la consulta, y al ver que sus expectativas no se cumplían, se manifestó el conflicto (económico) con la empresa minera. En la primera de estas comunidades no solo se determinó que los comuneros no trabajarían en la Empresa Minera Iscaycruz, sino que además se perjudicó las tierras cultivables durante la instalación del mineroducto, situación que colocó a la comunidad en total desventaja; y a pesar de que realizó continuos reclamos, no fue escuchada sino hasta que recurrió a amenazar con romper el mineroducto. Al constituirse la Empresa Minera Los Quenuales S.A. como nueva administradora de la unidad minera, la comunidad continuó sin tener oportunidades de trabajo en Iscaycruz, pero sí inició labores para la reparación de la tierra.

En cuanto a Mallay, cuando se realizó el trabajo de campo se halló que el conflicto por el uso y control de los recursos había empeorado por los constantes despidos de personal obrero y el ingreso en su reemplazo de personal calificado, pues ello afectaba las expectativas laborales planteadas al inicio de la relación. A raíz de esta segregación de personal, la comunidad se dividió en dos grupos: los que estaban a favor de la empresa porque recibían beneficios de ella, y los que estaban en contra de la empresa por no recibirlos.

En el caso de Huayllay, debido al contexto histórico que situaba a la comunidad en absoluta desventaja frente a la empresa minera, gran parte de los pobladores originarios migraron a Lima u otras ciudades del país por no tener oportunidades de trabajo en su pueblo, lo que 
hizo que un alto porcentaje de población foránea no solo ingresara a trabajar en la mina sino también a vivir en el pueblo. Es decir, los pobladores actuaron pasivamente y se retiraron de la zona, en lugar de exigir a la empresa oportunidades de trabajo (como se observa en el caso de Mallay, por ejemplo). Este movimiento, provechoso para las familias migrantes, fue sin embargo perjudicial para la comunidad, que ha ido perdiendo sus costumbres progresivamente e incorporando las de los foráneos.

\subsection{Proceso comunicativo en situaciones de conflicto minero}

Según la "teoría de la competencia comunicativa" (Habermas 1987), el fin de la comunicación es "estar de acuerdo", lo que es determinado por la comprensión recíproca y el saber participado entre los interlocutores. Para que la situación de "estar de acuerdo" consiga generar consenso ${ }^{14}$, los actores deben buscar superar la problematización surgida durante la acción comunicativa.

En el estudio de la comunicación entre empresas mineras y comunidades locales hay ciertas condiciones previas para lograr incluso la situación de "estar de acuerdo", debido a las diferentes características de los actores y al contexto de la relación entre ellos. Luego de explorar las teorías de comunicación relacionadas con el estudio, se ha graficado el proceso de comunicación entre la empresa minera y la comunidad, tomando como base el modelo interaccional de comunicación (Schramm 1954).

Como puede verse en el gráfico a continuación, la empresa y la comunidad aparecen como receptores y emisores a la vez, dado que la comunicación no es un proceso de una sola vía en el que uno emite mensajes y el otro solo los recibe, sino que ambas cosas suceden al mismo tiempo. Por ello se ha incorporado la presencia del ruido tanto al emitir el mensaje por la información que se filtra u omite, como al recibirlo y al dar el feedback.

${ }^{14}$ Definición de consenso basada en la "teoría consensual de la verdad" (Habermas 1987: 25). 
Este proceso comunicativo se desarrolla sobre la base de situaciones de conflicto ocurridas durante la historia de la relación que, junto a las diferencias entre los actores, constituyen dificultades para el consenso. Estas dificultades tienen lugar en condiciones de asimetrías económicas, sociales y políticas, y en aquéllas signadas por relaciones de poder.

La falta de información y de empoderamiento sobre sus derechos y de instrucción sobre minería son algunos de los factores que salen a la luz cuando empresas mineras con recursos económicos, manejo legal, última tecnología y acceso a información se instalan en comunidades con tantas carencias como las estudiadas.

\section{Grafico 1: Proceso comunicativo entre las empresas mineras y las comunidades locales}

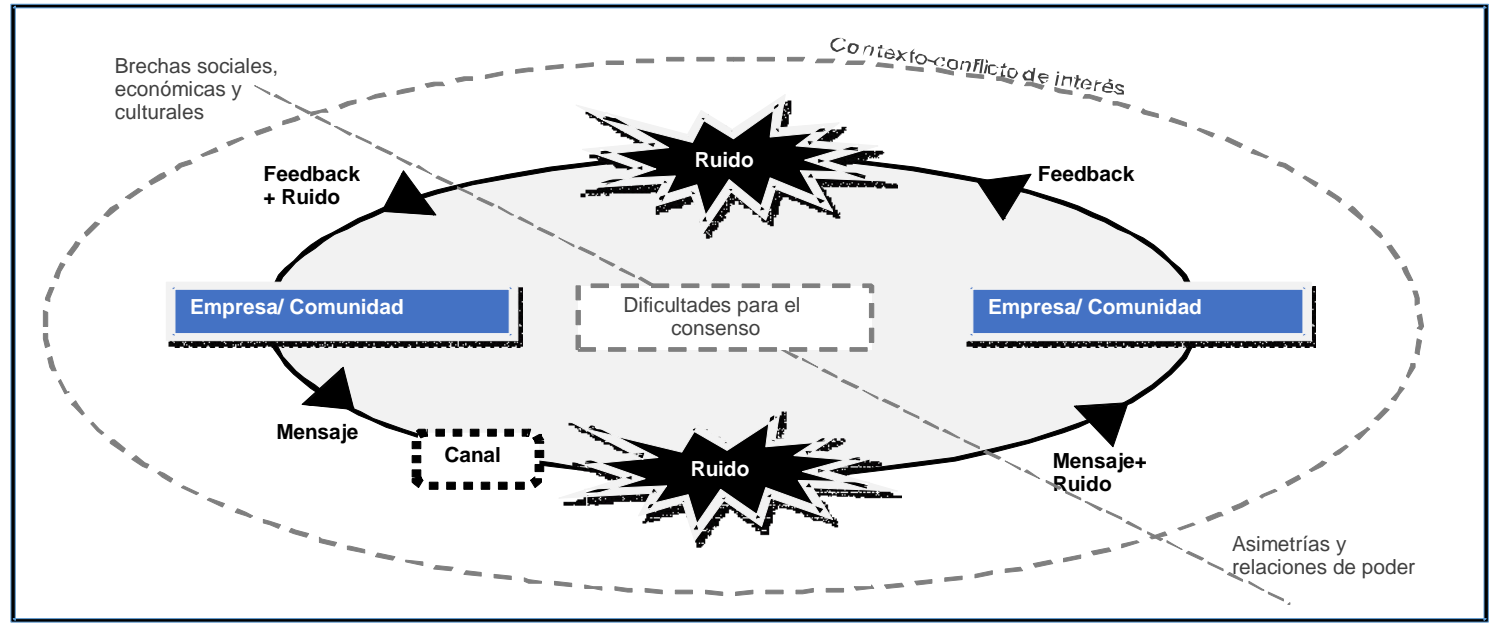

Elaboración propia.

Esta asimetría es muy visible en los casos de Mallay-Buenaventura y Curay-Los Quenuales, pues ambas son comunidades pequeñas y bastante pobres tanto por la cobertura de servicios básicos como por el acceso a información. En ambos casos ocurría que no solo los 
pobladores desconocían las reglas de juego establecidas y las condiciones que éstas generan, sino que, además, las autoridades estaban muy poco instruidas respecto de los derechos que les competen y tampoco buscan asesoramiento de un especialista.

Esta relación asimétrica entre la empresa minera y la comunidad local se torna dependencia cuando existe una relación laboral entre las partes, como ocurría en los tres casos estudiados, lo cual impedía que la comunidad planifique su desarrollo más allá de la presencia de la empresa minera. En dos de ellos las comunidades locales contaban con oportunidades de trabajo en las unidades mineras bajo la condición de mano de obra no calificada; pero estas oportunidades generaban una relación de dependencia que a la larga dificultaba el vínculo futuro.

Además, estas mismas condiciones hacen atractiva la comunidad local para otras poblaciones que se interesan en conseguir trabajo, pero esto genera un alto flujo migratorio: el ejemplo más representativo es Huayllay, cuyos habitantes originarios habían sido prácticamente desplazados por la población foránea, al punto de reducirse a menos del $50 \%$. Aquí, este proceso ha favorecido a la empresa minera, pues le ha permitido incrementar su influencia en la comunidad local y aminorar las posibilidades de conflicto.

En Mallay, a su vez, se daba un proceso muy interesante relacionado con las oportunidades de trabajo en la mina: muchos pobladores que migraron a Lima (capital de Perú) en busca de alternativas laborales habían retornado al pueblo y se habían reincorporado a la comunidad campesina para poder acceder al trabajo que brindaba el proyecto minero.

En lo que concierne a Curay, si bien se había firmado un acuerdo por el que la comunidad no tenía oportunidad de trabajo en la Unidad Minera Iscaycruz, el Convenio de Reparación de la Tierra implicaba la contratación de pobladores de esta comunidad. Dicha alternativa de empleo se convirtió en una salida de emergencia a la difícil situación por la que pasaban los comuneros debido a que la actividad que había sido el sustento económico tradicional por generaciones ya no presentaba las condiciones necesarias para su desarrollo, y tampoco 
habían adquirido otras capacidades para practicar otra actividad económica. Por el contrario, se evidenciaba que el convenio de reparación de la tierra firmado con la empresa había generado un fuerte lazo de dependencia económica de la comunidad hacia aquélla, vínculo ahora difícil de manejar.

En suma, en los tres casos estudiados, tanto las asimetrías en recursos, información y poder, como la relación de dependencia económica producto de las posibilidades de empleo en las unidades mineras, constituían factores determinantes para la definición de los actores en la relación. Así condicionados, cada parte construía un imaginario sobre la otra y sobre la identidad de esta otra en la relación.

La comunidad local es la más afectada en la construcción de identidad de los actores en la relación, ya que suele percibir a la empresa con alto nivel de responsabilidad sobre su futuro, lo que la hace adoptar una actitud pasiva respecto de su propio desarrollo. En los tres casos de estudio se encontró una relación de dependencia entre los actores, que impedía que la comunidad planifique su desarrollo más allá de la presencia de la empresa minera.

En el caso de Mallay, a pesar del poco tiempo transcurrido desde que la empresa se instaló en la zona y de la forma de relacionamiento construida, su presencia había repercutido en las organizaciones, que parecían más concentradas en las negociaciones con la empresa minera que en planificar un desarrollo a futuro.

En Curay, a su vez, la comunidad se había vuelto totalmente dependiente de la empresa. Cuando se realizó el trabajo de campo, la mayoría de comuneros subsistía casi exclusivamente con el salario que obtenía por las jornadas de reparación de la tierra. Este pago se interrumpió por el cierre de la unidad minera, y así los pobladores se quedaron sin sustento económico, pues no habían encontrado una actividad económica alternativa.

Los procesos descritos permiten comprender que la forma en que se construye la relación entre las empresas mineras y las comunidades campesinas implica el desarrollo de la identidad de los actores, y que se establece una relación de dependencia que tergiversa el 
vínculo y, a la larga, profundiza el conflicto. Esta relación de dependencia perjudica en mayor medida a la comunidad, pues ésta asume un rol pasivo en su desarrollo.

\section{Discusión: la intervención comunicativa desde la empresa}

A partir del estudio realizado, se ha podido constatar que, si bien los problemas de comunicación entre las empresas mineras y las comunidades locales no constituyen el origen de los conflictos, sí refuerzan el hecho de que la relación no marche y en algunos casos pueden convertirse incluso en el detonante para el estallido de una crisis. Tales problemas obedecen a una serie de deficiencias presentes en la base de la relación entre los actores, tanto en lo que atañe a la definición de éstos como a la ausencia de condiciones propicias para una buena relación.

Si bien cada conflicto responde a condiciones sociales, ambientales y económicas particulares, así como existen elementos estructurales y coyunturales, se pueden plantear modelos de intervención para la prevención de conflictos desde la empresa, el Estado y la sociedad civil. En este artículo, se propone una reflexión sobre las oportunidades de acción desde la empresa, dada la importancia que ha cobrado el sector minero para la economía nacional y el poder que ha adquirido en los últimos años.

Entre las tres categorías de actores, se puede reconocer que la empresa minera dispone de mayores recursos y capacidades para impulsar una cultura de la prevención, apuntando a la construcción de relaciones de confianza con todos los grupos de interés a los que genera impacto. Sin duda, ello implicará el replanteamiento de su cultura interna y el propio entendimiento de la responsabilidad social como un enfoque de gestión empresarial que implica el reconocimiento y la integración de las preocupaciones sociales, laborales y 
medioambientales de todos sus interlocutores (accionistas, empleados, clientes, proveedores, sociedad en general) en las operaciones que realiza.

Lo cierto es que, en los últimos años, se ha identificado una tendencia en las empresas mineras por comprender que la responsabilidad social va más allá del relacionamiento con la comunidad y que forma parte de la práctica gerencial de la empresa, lo que implica que la comunicación con los grupos internos y externos debe estar alineada. Buena parte de estos casos están relacionados con empresas de capitales extranjeros, que cuentan con lineamientos y estándares corporativos.

Dichos estándares se han globalizado y han contribuido, principalmente, al uso de tecnologías más limpias y un uso más eficiente de recursos por parte de las empresas del sector. La competencia por capitales de riesgo ha hecho que los inversionistas apliquen buenas prácticas corporativas y estándares más altos, y que por parte de la sociedad civil exista una mayor vigilancia sobre el tema, a través de las ONG.

Sin embargo, a la vez, hemos presenciado en los últimos años que buena parte de las empresas que declaran políticas de responsabilidad social con sus grupos de interés, se encuentran suscritas al Pacto Mundial y elaboran reportes de sostenibilidad siguiendo los lineamientos del Global Reporting Initiative, han venido estando involucradas con casos de conflicto social con las comunidades de su entorno.

Ello nos permite constatar que, las empresas mineras no han logrado aún gestionar el relacionamiento con sus grupos de interés y sobre todo comunicar de manera adecuada la implementación de los nuevos estándares adoptados. Como se ha podido apreciar a través de los tres casos estudiados, la comunicación ha estado dirigida hacia la comunidad, básicamente a través de la implementación de programas sociales, guardando poca coherencia con el relacionamiento con otros grupos de interés importantes, como son los colaboradores y los proveedores. 
Ello ha permitido que se consolide una percepción negativa en la opinión pública que no se condice con el mencionado éxito económico alcanzado por el sector. En primer lugar, se piensa que la minería contamina el ambiente, haciéndola incompatible con otros sectores económicos como, por ejemplo, la agricultura. En segundo lugar, se asume que, al tratarse de una industria primaria, no genera valor agregado en la economía local. En tercer lugar, se la percibe como un sector que no genera impacto en el desarrollo de las comunidades que viven en su entorno. $Y$ finalmente, se cree que no actúa con transparencia, ya que se sabe poco de sus operaciones y resultados (Cantuarias, F., 2010).

Generar una buena reputación sobre la base de las percepciones descritas implica que el sector esté llamado a comprender la importancia de comprometerse con todos sus grupos de interés, lo cual supone, entre otros aspectos, respetar los derechos humanos, proveer beneficios a sus trabajadores y en las comunidades donde opera, y conducir el negocio de manera ética, justa y transparente. Ello supone, entonces, el alineamiento con los públicos internos (geólogos, gerentes, obreros, etc.) y comunicar los logros hacia afuera de manera efectiva, lo cual constituye un reto para el sector.

En el siguiente gráfico se expresa el flujo de comunicación de la empresa hacia sus grupos de interés a través de las áreas interlocutoras: Recursos Humanos (RR.HH.), Logística, Relaciones Comunitarias (RR.CC.), Comercial, Gerencia General y Asuntos Corporativos. Dicha comunicación aislada por parte de los interlocutores presenta el riesgo de emitir mensajes distintos a los grupos de interés, cuyos miembros pueden coincidir y compartir información.

Si bien se reconoce que la comunidad constituye un grupo de interés crítico para una empresa minera, con el cual se deben planificar las acciones de comunicación, resulta fundamental que la empresa pueda generar un equilibrio con los otros grupos de interés, sobre todo considerando que los colaboradores y proveedores pueden pertenecer a la comunidad también. 
Dado que existen multitud de aspectos que comunican sobre la empresa, es importante planificar cada uno de ellos, buscando coherencia entre todos los recursos y mensajes utilizados. Las empresas que carecen de un plan de comunicación general pueden presentar la fragmentación en la comunicación, siendo posible que cada área realice acciones aisladas que no guarden relación entre sí (Marín, F., 2008).

Sin embargo, es importante reconocer que, nos encontramos en un sector con alta complejidad que presenta dificultades a su interior para poner en práctica el mencionado alineamiento, pues supone construir indicadores y estrategias de relacionamiento, y propone fortalecer las competencias de los interlocutores responsables del relacionamiento con los grupos de interés, para su puesta en práctica y monitoreo participativo.

\section{Gráfico 2: Comunicación entre la empresa extractiva y sus grupos de interés}

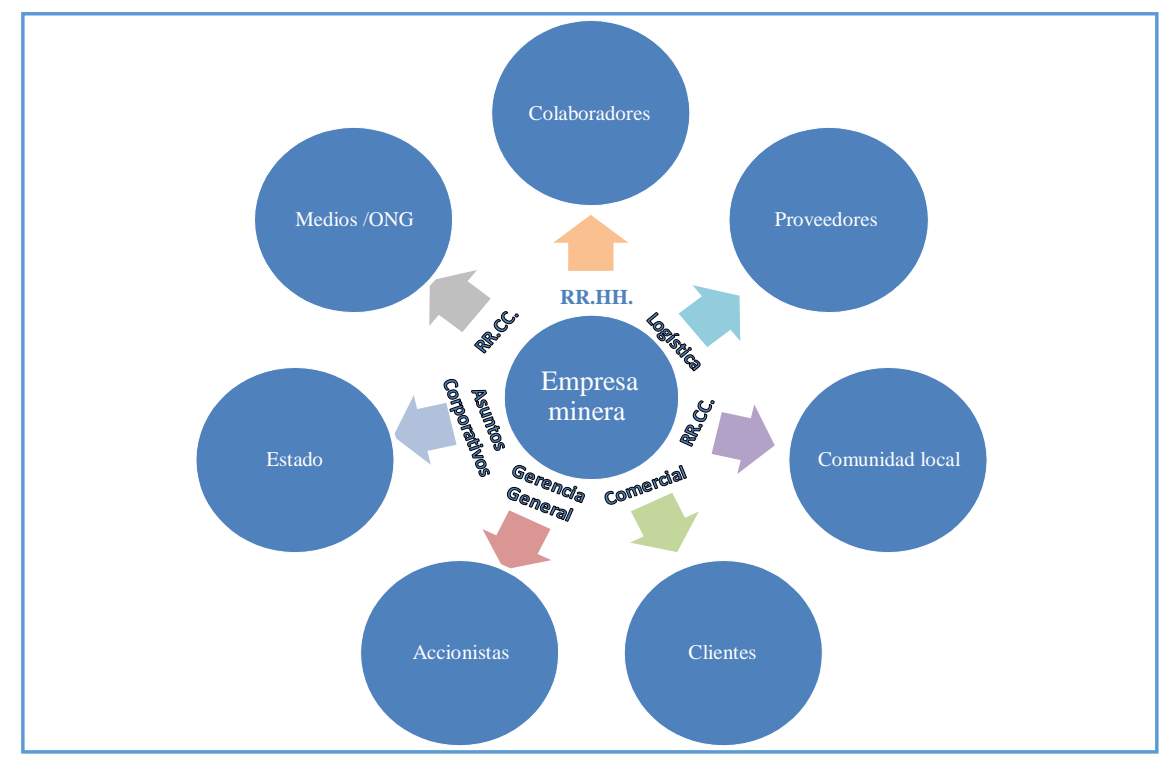

Elaboración propia 
Ello se debe a que, conseguir llevar a la práctica la RSC supone generar un verdadero compromiso empresarial desde la dirección hasta los obreros, lo cual implica enfrentar la escasa formación en el tema y reconocimiento de su importancia.

Todo ello implica mejorar la comunicación interna, la transferencia de estándares hacia los proveedores, implementar prácticas de transparencia con los accionistas y clientes, contribuir al fortalecimiento de capacidades del Estado y generar una estrategia de comunicación socio-ambiental dirigida a la comunidad. De esta forma, se asegura una coherencia entre los mensajes emitidos hacia cada uno de los grupos de interés, lo que permitirá construir confianza y gestionar las externalidades sociales, ambientales y económicas con cada uno, y a su vez generará un impacto positivo en la reputación de la empresa.

Partiendo de esa premisa, el gráfico 2 propone un modelo de relacionamiento que parte del alineamiento interno en la empresa, sobre la base de un plan general de comunicación y relacionamiento con los grupos de interés. El plan de comunicaciones debe partir de la identificación de las necesidades comunicativas de cada uno de los grupos de interés, establecer los objetivos, el mensaje a comunicar y las acciones necesarias. De esta manera, la organización logra una mayor coherencia en la comunicación y obtiene una sinergia entre las diferentes acciones.

\section{Gráfico 2: Modelo de relacionamiento con los grupos de interés}

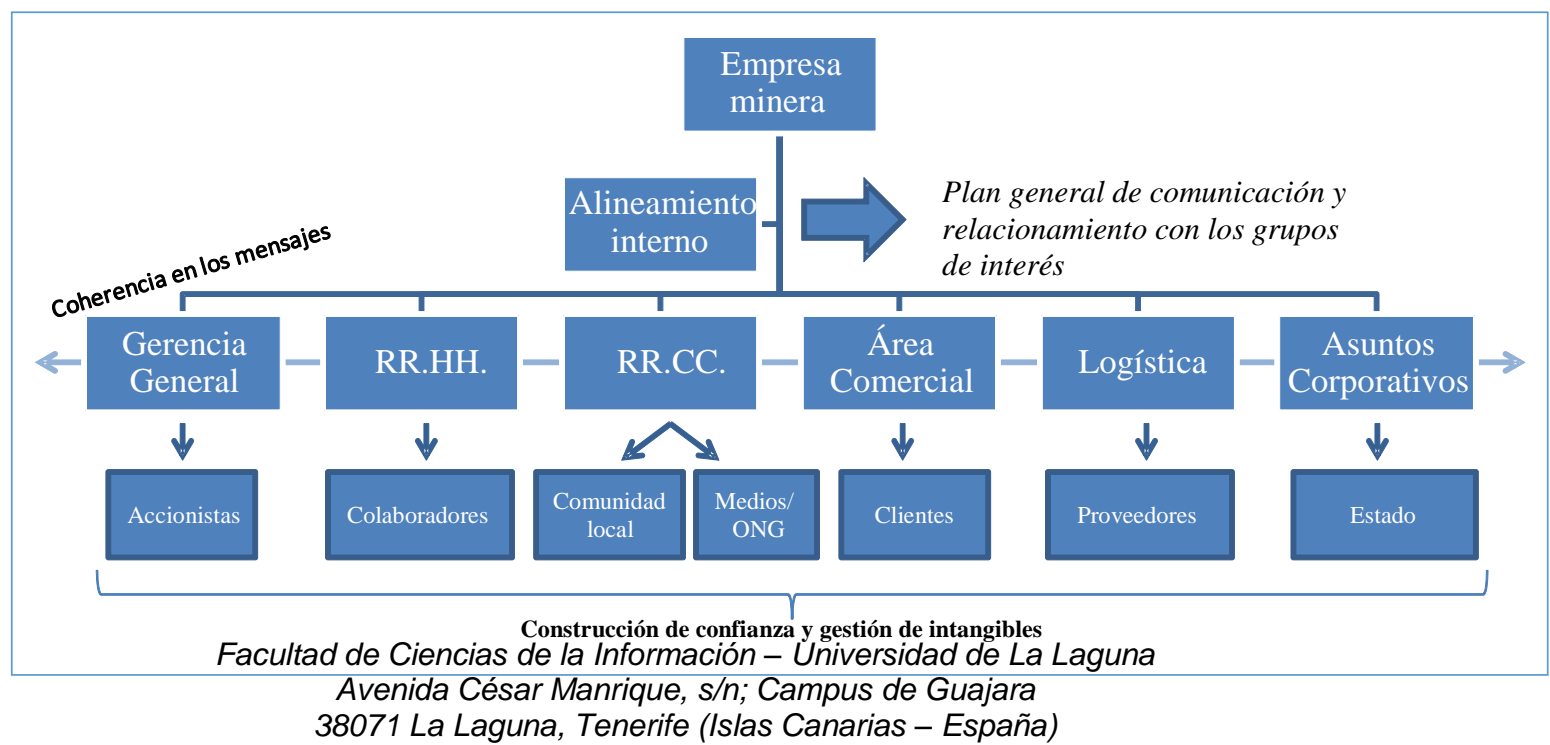




\section{Elaboración propia}

Las empresas que sepan integrar adecuadamente la RSC en su estrategia, y posteriormente en el nivel operativo, tendrán grandes posibilidades de conseguir ventajas competitivas que les faciliten un mejor posicionamiento estratégico. Los resultados obtenidos del diálogo con los grupos de interés se integran en el proceso de creación de valor de la empresa: se genera una mayor motivación y productividad por parte de los empleados, mayor nivel de satisfacción y lealtad de los clientes / usuarios y proveedores, construcción de relaciones de confianza con el Estado, y un mayor reconocimiento de la sociedad civil. A su vez, todo ello contribuye a la gestión de la reputación de la empresa.

Sin embargo, se ha comprobado que, el establecimiento de un sistema de gestión en una organización no es nada sencillo. Por encima de las formas, procesos, y herramientas a utilizar, existe una máxima a la hora de implementar la RSC: priorizar la gestión interna sobre la gestión externa. Es decir, antes de comunicar a los grupos de interés las acciones realizadas, debemos lograr que la organización haya integrado la RSC a nivel interno (Ancos, H., 2010).

Esta máxima es la que provoca que, dentro de la organización, la idea de implementar un sistema de gestión de RSC nazca con claridad y convencimiento por parte de todos, pero sobre todo con el compromiso de la alta dirección para llevarlo a cabo. Efectivamente, el compromiso de los directivos con la sostenibilidad y su capacidad para integrar estas acciones y programas dentro de su estructura empresarial, constituye un favor clave para el diseño y buen desempeño empresarial de una estrategia de RSC. 
Es vital que la alta dirección trabaje para integrar la estrategia de la RSC junto con las diferentes estrategias de la compañía, y que forme parte de la estrategia general de la compañía. Asimismo, la alta dirección de la organización deberá comunicar su compromiso al resto de empleados, en el marco de su política de RSC.

Ahora bien, poner en práctica los lineamientos es un proceso complejo, pues supone un cambio profundo que necesita de nuevas ideas y recursos. Frente a ello, puede encontrarse resistencia al cambio y otro tipo de dificultades para la aplicación de la responsabilidad social corporativa. Por ello, resulta importante que exista una convicción interna y una determinación real, ya que de esta forma no se dudará en dedicar todos los recursos necesarios, mediante un proceso de aprendizaje y mejora.

Para conseguir involucrar a toda la organización debemos superar dos obstáculos: el escepticismo y el desconocimiento de la responsabilidad social corporativa. Normalmente puede haber un rechazo derivado de su percepción como una carga de trabajo extra, porque se considera que utiliza los recursos necesarios para otras funciones, o porque se tiene una imagen viciada y negativa de la RSC, etc.

Ello implica, entonces, gestionar un cambio en la cultura de la empresa, ya que a menudo los proyectos de RSC pueden requerir el trabajo de varios equipos multidisciplinares y multidepartamentales conjuntamente, cosa que normalmente choca con la forma de trabajar de la mayoría de las empresas (Ancos, H., 2010).

Tras el compromiso de la dirección, la ejecución de una estrategia de RSC ha de comenzar con el análisis interno u autodiagnóstico. El siguiente paso será la captura de información sobre las percepciones de sus grupos de interés en relación con todas las acciones y operaciones de la empresa y en relación con la denominada "Triple Bottom Line" o triple línea básica que alude a la consecución de los objetivos económicos, sociales y medioambientales, promoviendo un espacio de discusión con los grupos de interés. 
El tercer paso será la fase de análisis de esta información y la elaboración de un plan de actuación en RSC que se alinee con la estrategia corporativa. Para ello, habrán de elaborarse indicadores que orienten la actividad y la evolución de la empresa en las distintas áreas de trabajo en un proceso de mejora continua. La cuarta fase se corresponderá con la implementación de dicho plan, y finalmente, habrá de prestarse atención a los procesos de control del desempeño.

La comunicación desempeña un rol fundamental en todo el proceso descrito, desde la identificación y mapeo de los grupos de interés, hasta el diseño, implementación y monitoreo de la estrategia de relacionamiento con cada uno. Ello implica que, si bien el diagnóstico y gestión de la RSC se aborda desde perspectivas legales, económicas, sociológicas, comunicativas, entre otras; la comunicación constituye un aspecto clave en el manejo de herramientas para la integración de la RSE a la gestión y cultura empresarial, y para la medición y evaluación de su impacto.

Es así que, la comunicación ha dejado de ser concebida como una herramienta empleada con fines utilitarios, para revalorar su potencial estratégico. Comunicar el desarrollo ético y sostenible hacia los clientes y accionistas, establecer canales apropiados de diálogo con cada uno de los grupos de interés, hacer llegar los mensajes correctos a los interlocutores, entre otros, han dejado de ser acciones esporádicas y aisladas para constituir una estrategia de valor en las principales empresas en crecimiento.

\section{CONCLUSIONES}

A partir del estudio realizado, se ha podido constatar que los conflictos entre empresas mineras y comunidades locales se deben a un desencuentro de intereses, formas de vida, brechas sociales y asimetrías que se encuentran en la base de la relación y que dificultan la existencia de condiciones propicias para el entendimiento mutuo. Las prácticas de comunicación pueden reforzar el hecho de que la relación no marche y en algunos casos pueden convertirse incluso en detonante para el estallido de una crisis. 
La debilidad en la definición de los actores ha sido uno de los principales obstáculos identificados. En los casos estudiados se encontró no sólo personajes que cumplían la función de representantes de las comunidades campesinas sin velar por los intereses de éstas, sino también la ausencia de objetivos colectivos claros y armónicos que guíen su actuación. Por su parte, la empresa minera, en principio, cuenta con mecanismos formales para definir quién habla y qué dice; sin embargo, se ha podido identificar que dichos mecanismos no son del todo efectivos.

Los términos de la relación entre la empresa y la comunidad se vinculan en gran medida al rol del Estado, que ha privilegiado sus políticas de promoción de la inversión sobre los parámetros de regulación del sector, dificultando su participación imparcial en situaciones de conflicto y profundizado la asimetría de poder entre la empresa y la comunidad. Asimismo, si bien la participación del Estado en las situaciones de conflicto no se da de la misma manera en todos los casos, se observó que su accionar ha estado más orientado a resolver problemas generados por los estallidos de conflictos que a identificar y atacar sus causas.

Con respecto a los temas de fondo de los conflictos, si bien se encontró en las tres comunidades una fuerte preocupación por el impacto ambiental de la actividad minera, es importante señalar que la población se encontraba inicialmente a favor de la inversión minera y aspiraba a que ésta dinamice la economía local a través de la creación de empleo y negocios relacionados. Muchos de los conflictos encontrados se originaban cuando no se veía concretar las expectativas sobre los posibles beneficios de la actividad, lo cual se exacerbaba si un eventual problema ambiental comprometía las actividades agropecuarias que constituían el sustento familiar.

También se evidenció, que al comienzo de la relación entre la empresa y la comunidad el conflicto de intereses es entendido de diferente manera por los actores, lo cual obedece a un desencuentro de valores y modos de vida entre las partes. Por ello, pese a las acciones que la empresa realiza para ablandar el descontento de la comunidad, se generaban tensiones 
que, acumuladas con el descontento y resentimiento de la comunidad, podían transformarse en crisis si no se gestionan de manera adecuada.

Si bien se trata de pares en la formalidad, en la práctica, las empresas mineras tienen una serie de ventajas sobre las comunidades locales, las cuales favorecen una relación de poder entre ambas partes que condicionará el proceso comunicativo necesario para la toma de decisiones. Ello se fundamenta en las asimetrías de comunicación relacionadas al conocimiento de las reglas y asuntos en juego. Este asunto es clave, sobre todo al inicio de la relación, ya que las comunidades por lo general no manejan información respecto de las leyes y los derechos que las amparan por ser propietarias de la superficie de la tierra que se explotará.

En este marco, existen múltiples escenarios de intervención para la prevención de conflictos. Por el lado de la empresa, se ha identificado una alta potencialidad en la construcción de relaciones de confianza con sus grupos de interés. De hecho, se reconoce que la empresa minera dispone de mayores recursos y capacidades para impulsar una cultura de la prevención; sin embargo, ello implicará el replanteamiento de su cultura interna y el propio entendimiento de la responsabilidad social como un enfoque de gestión empresarial que implica el reconocimiento y la integración de las preocupaciones sociales, laborales y medioambientales de todos sus interlocutores (accionistas, empleados, clientes, proveedores, sociedad en general) en las operaciones que realiza.

Si bien en los últimos años se ha podido identificar una tendencia en las empresa mineras por comprender que la responsabilidad social va más allá del relacionamiento con la comunidad y que forma parte de la práctica gerencial de la empresa, a la vez hemos presenciado que buena parte de éstas han venido estando involucradas con casos de conflicto social con las comunidades de su entorno. A partir de ello, se ha constatado que, las empresas mineras no han logrado aún gestionar el relacionamiento con sus grupos de 
interés y sobre todo comunicar de manera adecuada la implementación de los nuevos estándares adoptados.

Partiendo de ello, se ha propuesto un modelo de relacionamiento que parte del alineamiento interno en la empresa, sobre la base de un plan general de comunicación y relacionamiento con los grupos de interés. De esta forma, se asegura una coherencia entre los mensajes emitidos hacia cada uno de los grupos de interés, lo que permitirá construir confianza y gestionar las externalidades sociales, ambientales y económicas con cada uno, y a su vez generará un impacto positivo en la reputación de la empresa.

La comunicación desempeña un rol fundamental en la puesta en práctica de dicho modelo, desde la identificación y mapeo de los grupos de interés, hasta el diseño, implementación y monitoreo de la estrategia de relacionamiento con cada uno. Ello implica que, si bien el diagnóstico y gestión de la RSC se aborda desde perspectivas legales, económicas, sociológicas, comunicativas, entre otras; la comunicación constituye un aspecto clave en el manejo de herramientas para la integración de la RSE a la gestión y cultura empresarial, y para la medición y evaluación de su impacto.

\section{BIBLIOGRAFÍA}

AccountAbility (2006) De las palabras a la acción: El compromiso con los stakeholders. Manual para la práctica de las relaciones con los grupos de interés. Recuperado el 04 de abril de 2013, de: http://www.accountability.org/images/content/2/0/204.pdf

Alayza, A. (2007). No pero sí. Comunidades y minería: consulta y consentimiento previo, libre e informado en el Perú. Lima: Oxfam.

Aste, J. (2004a). Procesos de concertación en zonas mineras en el Perú. Lima: GRADE. 
Aste, J. (2004b). Resolviendo conflictos entre el Estado, las empresas mineras, las comunidades campesinas y los organismos de la sociedad civil: procesos de concertación en zonas mineras del Perú. Lima: GRADE/ECO.

Ancos, H. (2010) La Responsabilidad Social Corporativa y sus Actores: Mitos y Desafíos de la RSC. Madrid: Instituto Complutense de Estudios Internacionales.

Barnechea, A. Y Tumi, F. (2008). El Síndrome del Cuarto del Rescate. Entrevistas a expertos, empresarios, autoridades y líderes sociales sobre la relación del Perú con sus industrias extractivas. Lima: Paraca Comunicaciones.

Barrantes, R. (2005). Te quiero pero no»: minería, desarrollo y poblaciones locales. Lima: IEP/OXFAM.

Cantuarias, F. (2010) La Responsabilidad Social en la minería: un avance. Lima: Sociedad Nacional de Minería Petróleo y Energía. Recuperado el 20 de febrero de 2013, de: http://www.solomineria.com.pe/html/responsa/pdf/antamina/responsabilidadsocial.pdf

Carrillo, S. (2011). Comunidades y Minería: la Comunicación en el Conflicto. Tres estudios de caso en la sierra central del Perú. [Tesis de licenciatura] Lima: Pontificia Universidad Católica del Perú.

Castillo, G. (2006). "Se vende oro: La creación de espacios contestados en la promoción de la minería". En Mirando la esfera pública desde la cultura en el Perú. Lima: CONCYTEC.

Castillo, P. et al. (2007). "Las comunidades campesinas en el siglo XXI: balance jurídico". En ¿Qué sabemos de las comunidades campesinas? Lima: Allpa Comunidades y Desarrollo.

Centro de Estudios del Cobre y la Minería (2012). Tendencias de la Exploración Mundial. Nueva Scotia: Metals Economics Group. 
Consejo Internacional de Minería y Metales (2008). Perú: desafío de la riqueza mineral: utilizar la dotación de recursos para impulsar el desarrollo sostenible. Génova: Conferencia de las Naciones Unidas sobre Comercio y Desarrollo/Grupo Banco Mundial.

Consejo Internacional de Minería y Metales (2008). Annual Review 2012. London: ICMM.

Cooperacción (2013). XII Informe del Observatorio de Conflictos Mineros en el Perú. Lima: Cooperacción.

De Echave, J. (2009). Minería y conflicto social. Lima: IEP/Centro Bartolomé de Las Casas.

Defensoría del Pueblo (2004-2013). "Reporte, conflictos de distinta intensidad entre población y entidades públicas conocidos por la Defensoría del Pueblo". Disponible en: $<$ http://www.defensoria.gob.pe/conflictos-sociales-reportes.php $>$

Diez, A. (1999). "Organizaciones de base y gobiernos locales rurales: Mundos de vida, ciudadanía y clientelismo". En Repensando la política en el Perú. Lima: Red para el Desarrollo de las Ciencias Sociales en el Perú.

Diez, A. (2007). «Organización y poder en comunidades, rondas campesinas y municipios». En ¿Qué sabemos de las comunidades campesinas? Lima: Allpa Comunidades y Desarrollo.

Gumucio, A. (2004). El cuarto mosquetero: la comunicación para el cambio social. Barranquilla: Investigación y Desarrollo. Disponible en: $<$ http://redalyc.uaemex.mx/redalyc/src/inicio/ArtPdfRed.jsp?iCve=26800101>.

Habermas, J. (1987). Teoría de la acción comunicativa. Madrid: Taurus.

Macroconsult (2012). Impacto económico de la minería en el Perú. Lima: SNMPE.

Revesz, B. Y Alejandro D. (2006). "El triángulo sin cúpula (o los actores desregulados en los conflictos sociales)". En Perú hoy: nuevos rostros en la escena nacional. Lima: Desco. 
Porter, M. y Kramer, M. (2011). Creating Shared Value. I low to invent capitalism -and unleash a wave of innovation and growth. Harvard Business Review, enerofebrero, pp.117.

Presidencia del Consejo de Ministros de la República del Perú - PCM (2013). Informe de diferencias, controversias y conflictos sociales № 05 - Abril 2013. Lima: Oficina Nacional de Diálogo y Sostenibilidad - ONDS. Disponible en: http://www.pcm.gob.pe/transparencia/willaqniki/willaqniki05.pdf

Rodríguez, C., Obregón, R. y Vega, J. (2002). Estrategias de comunicación para el cambio social. Quito: Friedrich-Ebert-Stiftung, Proyecto Latinoamericano de Medios de Comunicación.

Schramm, W. (1954). Process and effects of mass communication. Urbana: University of Illinois Press.

West, R. (2005). Teoría de la comunicación: análisis y aplicación. Madrid: McGraw Hill.

\section{Forma de citar este artículo en bibliografías}

CARRILLO HOYOS, S. (2013): "El valor de la comunicación estratégica para la gestión responsable y la prevención de conflictos mineros", en Revista PANGEA, 4, páginas 325 a 360. Red Académica Iberoamericana de Comunicación. Recuperado el de de 2 de: http://revistapangea.org 